\title{
Correction to: Unilateral freezing of gait or "magnetic feet phenomenon" caused by ischemic lesion involving fronto-striatal networks
}

\author{
Alberto Lerario $^{1} \cdot$ Floriano Girotti $^{1} \cdot$ Jenny Sassone $^{2,3} \cdot$ Barbara Poletti $^{1} \cdot$ Andrea Ciammola $^{1} \cdot$ Vincenzo Silani $^{1,4}$ \\ Published online: 3 July 2021 \\ (c) Fondazione Società Italiana di Neurologia 2021
}

Correction to: Neurological Sciences (2021)

https://doi.org/10.1007/s10072-021-05290-3

The original article contains an error in author name. Given name and family name has been interchange. The correct presentation of author names are presented above.

The original article has been corrected.

Publisher's note Springer Nature remains neutral with regard to jurisdictional claims in published maps and institutional affiliations.

The original article can be found online at https://doi.org/10.1007/ s10072-021-05290-3.

Alberto Lerario

alberto.lerario@gmail.com

$\triangle$ Andrea Ciammola

a.ciammola@auxologico.it

1 Department of Neurology and Laboratory of Neuroscience, IRCCS Istituto Auxologico Italiano, Piazzale Brescia 20, 20149 Milan, Italy

2 San Raffaele Scientific Institute, Via Olgettina 58, 20132 Milano, Italy

3 Vita-Salute San Raffaele University, Via Olgettina 58, 20132 Milano, Italy

4 Department of Pathophysiology and Transplantation, Dino Ferrari" Center, Università degli Studi di Milano, Via Francesco Sforza 35, 20122 Milan, Italy 